\title{
The construction of English verb "send": A cognitive linguistics study
}

\author{
Moch. Panji Prabaskoro ${ }^{a, 1, *}$, Prayudha ${ }^{\mathrm{b}, 2}$ \\ ${ }^{\text {a,b }}$ Universitas Ahmad Dahlan, Jl. Ringroad Selatan, Kragilan, Tamanan, Kec. Banguntapan, Bantul, Daerah Istimewa Yogyakarta 55191, Indonesia \\ ${ }^{1}$ panjiprabaskoro@gmail.com*; ${ }^{2}$ prayudha@pbi.uad.ac.id \\ * corresponding author
}

\section{ARTICLE INFO}

Article history

Received 17 December 2019

Revised 22 March 2020

Accepted 14 August 2020

Available Online 15 January 2021

Keywords

construction

cognitive linguistic

English verb

send

\begin{abstract}
This research is entitled "The Construction of English Verb "SEND": A Cognitive Linguistic Study". It is based on cognitive linguistic, because with cognitive linguistic it could be seen how exactly the construction of English verb "send" and how the construction could have that construction. The aims of the research are; 1) to analyze the construction of English verb "SEND" as a single verb; and 2) to analyze the Construction of English verb "SEND" as a phrasal verb. The data will be collected by using observation method and noting technique. Then, it will be analyzed by using distributional method with expansion, ellipsis, and immediate constituent division. All data that have been collected and analyzed will then be described by qualitative method. As a result, there would be two main outcome found in this research. First, the construction of English verb "send" in the form of single verb, those are English verb "send" as single verb in intransitive construction, transitive construction and ditransitive construction. Second, the construction of English verb "send" in the form of phrasal verb, those are English verb "send" as phrasal verb in intransitive construction, transitive construction, and ditransitive construction.
\end{abstract}

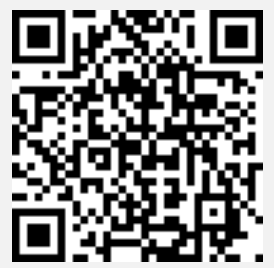

This is an open access article under the CC-BY-SA license.

\section{Introduction}

Constructions are taken to be the basic units of language, which is a group of language unit or symbolic unit with meaning (Goldberg, 1995: 4). In cognitive linguistics, grammatical construction is viewed as a continuous network between the symbolic elements. The continuity occurs as the result of the relation between the verb and its argument. Verhaar (2010: 193) stated that the semantic role of a verb lexically would affect the character of its argument. The character of the argument that is affected by the lexical feature of the verb are i) verb valence, ii) role of the argument, iii) character of the argument (persona. total, type, and case). Cognitive linguistics is considered as the appropriate approach to discuss more in-depth research about polysemy as its characteristic which observes language more broadly and comprehensive. Cognitive linguistics try to rearrange the finding of linguistic theory that exist into generalisation commitment and cognitive commitment.

The discussion of polysemy SEE found in the research of Gisborne (2010) discover a new insight in polysemy study. The research used the approach models which analyze the grammatical structure of lexical polysemy. The results shows that other than 15 extensions meaning, the approach also found that the construction of ditransitive make the meaning of SEE change. The other approach model was also be used in the research of Prayudha (2014) in analyzing the construction of 
polysemy verb LOOK. The result shows the construction of polysemy verb SEE in three different form; 1) LOOK as single verb, 2) LOOK as phrase verb, and 3) LOOK in idiomatic construction.

The result of the data observation in Oxford Dictionary (Oxford Advanced Learner's Dictionary), verb "SEND" appear to be in these constructions:

1) Its use would send out the wrong signal to consumers

2) Her music always sends me to sleep.

The data above show us the construction of "send" that appear in different construction. Data (1) appears in ditransitive construction with a phrasal verb "Send out", while data (2) appears in transitive construction with a single verb "send". Therefore, the study will focus on analyzing the construction of the English verb "send" as a single verb and phrasal verb.

Lakoff (1990 in Evans, Bergan, \& Ziken, 2007: 4) explained that cognitive linguistic has a number of key commitments. Evans \& Green (2006: 27-48) divide the commitments into Generalization Commitment and Cognitive Commitment. Based on both commitment explained above about cognitive linguistic, the consequences that arise are there can be found two field of study in cognitive linguistic those are cognitive semantic and cognitive approach to grammar (Evans \& Green, 2006: 48).

One of the research model of cognitive approach to grammar is Construction Grammar by Fillmore and Paul Kay, Adle Goldberg, and also William Croft. The role of construction grammar is to study the comprehensive relationship between semantic and syntax and to show how close the two systems are (Gisborne, 2010: 63-65). Both of the systems are exist in the discussion of argument linking.

Argument linking - which become the main core of construction grammar - relate with verb classification and semantic role. Chafe (1970: 95-104) distinguish verb in three types which are state verb, process verb, and action verb. Those verb types then brought down to a number of other verb types. Those verb types are action-process verb and experimental verb. Jackendoff (in Van Vallin 2005: 53), distinguish semantic role of the arguments in accordance with the characteristic of the verb. He describe the relation between verb type.



Fig. 1. Continuum from verb-specific semantic roles to grammatical relations.

\section{Research Method}

The research method used in this study belongs to the qualitative method which affiliates with descriptive research. Qualitative research is focused on analyzing and gathering the informative data presented in document, interviews, etc. (Moyer, 2008; 27). Following Tavakoli $(2012 ; 151)$, data in the form of words such as descriptions, observations, impressions, and recordings include in 
qualitative data. The descriptive method is used as the data found in this research will be analyzed and explained in the form of description.The data used in this study is sourced from dictionary and corpus. Dictionary is used as the main source data as it gives a systematic data. The data from corpus is added as it can provide more data reference.

The research would use observation method with noting technique by Sudaryanto (2015) in collecting the data. Observation method is used by observing, which is by observing the use of the language. Hence, this method could be parallelized with Observation and Monitoring method in social study, especially anthropology (Sudaryanto, 2015; 203). Sudaryanto $(2015 ; 206)$ stated that noting technique could be done by certain writing tools. The exact steps in collecting the data are as follows: Go to Oxford Advanced Learner's Dictionary and start observing the data

- Copies the data sourced from Oxford Advanced Learner's Dictionary to Microsoft word.

- Grouping the data obtained from the dictionary based on their construction (such as; single verb or phrasal verb) and (such as; send, send forth, send for, etc.)

- Go to BNC site Page and observe the data

- Enter keyword "SEND" and collect data that based on the group of data found in Oxford Dictionary (such as; single verb or phrasal verb) and (such as; send, send forth, send for, etc.)

- The collected data from BNC is then copied to Microsoft word and group the data based on the construction of the Verb "SEND."

- The data from Oxford Dictionary is signed by label Ox, while the data from BNC is labeled by BNC.

This study used Agih method, Agih method by Sudaryanto (2015: 18) is defined as an analysis method where the determinant is part of the concerned language itself. To analyze the construction of English verb "SEND," this research used ellipsis and expansion technique to find how the verb "Send" can or cannot turn into another construction, and whether the sentence can be intransitive, transitive or ditransitive. This research also used immediate constituent division techniqueto analyze the construction of the sentence found in Oxford and BNC. The technique called as immediate constituent division because the technique divides the element or the data into several parts (Sudaryanto, 2015; 37).

\section{Findings and Discussion}

This section would explain the findings with the aim of the research which are; 1) to analyze the construction of English verb "SEND" as a single verb; and 2) to analyze the Construction of English verb "SEND" as a phrasal verb.

\subsection{English Verb "SEND" as a Single Verb}

As a single verb, the verb "send" will be classified into three classifications. Verb "send" in intransitive construction, verb "send" in transitive construction, and verb "send" in ditransitive construction.

\section{1) SEND in Intransitive Construction}

The verb "send," as a single verb could not occur in intransitive construction as the verb "send" as a single verb will always need an object in the sentence. So verb "send" as a single verb can only occur in transitive and ditransitive construction. Even if the English verb "send" could not occur in intransitive construction, there will be some cases when verb " send" could seem to be an intransitive construction while they are not in intransitive construction. The example could be seen as follows:

(1) A radio signal was sent to the spacecraft. Ox

$$
\text { S: Ptn SV:APV Goal }
$$

(2) We may have some surplus to send. BNC

$$
\text { S: Ag Aux SV:SV O: Sml Goal }
$$


Both data above might seem to be in intransitive construction as it has no object coming after the English verb 'send', but they are not. Data (1) is in transitive construction because data (1) is in the form of passive voice. Passive voice can only be formed by a transitive verb and not by an intransitive verb since intransitive verb does not have an object to begin with.Data (2) is actually in transitive construction because the verb "send" in the sentence does not work as the main verb of the sentence but as to-infinitive. To-infinitive is a verb with the addition of preposition to that could act as an adjective, noun, and adverb in a sentence.

\section{2) SEND in Transitive Construction}

Verb "send" in transitive construction in this research will be divided into four categories. First, verb "send" in transitive construction with the pattern (Agent+Send+Patient). Second, verb "send" in transitive construction with the pattern (Agent+Send+Patient+Goal). Third, verb "send" in transitive construction with the pattern (Agent+Send+Patient+Complement). Last, verb "send" in transitive construction that is manipulated with ellipsis and expansion technique.

a) Agent+Send+Patient

The first grammatical construction is (Agent+Send+Patient).

(3) I will send a car. BNC

S:Ag Aux SV:AVP O: Ptn

(4) I cannot send this letter. BNC

S:Ag Aux SV:APV O:Ptn

(5) You didn't send any noodles. BNC

S:Ag Aux SV:AVP O:Ptn

(6) My parents send their love. Ox

S:Ag SV:APV O:Ptn

In this case, the verb "send" would need two arguments where the first argument will function as the subject with a semantic role as an agent and the second argument will function as an object with a semantic role as a patient.

\section{b) Agent+Send+Patient+Goal}

The pattern in this grammatical construction is (Agent+Send+Patient+Goal).

(7) They will probably send me to prison. BNC

S: Ag Aux Adv SV:APV O:Ptn Goal

(8) I will send them to the plane. BNC S:Ag Aux SV:AVP O:Ptn Goal

(9) I have sent Tom to buy some milk. Ox S:Ag SV:APV O:Ptn Goal

The verb "send" would be having two arguments consist of a subject with the semantic role as agent and object with the semantic role as a patient. Then, at the end of the sentence, there would be additional information where this additional information would have a semantic role as a goal.

c) Agent+Send+Patient+Complement

The pattern of this grammatical construction would be (Agent+Send+Patient+Complement).

(10) The results of his discovery send him mad. BNC

$$
\mathrm{S}: \mathrm{Ag} \quad \mathrm{SV}: \overline{\mathrm{APV}} \overline{\mathrm{O}: \mathrm{Ptn}} \overline{\mathrm{Cpl}}
$$

(11) She sent the letter by airmail. Ox

$$
\text { S: Ag SV:APV O:APV Cpl }
$$

(12) We will send all the details with the catalogue. BNC

$$
\text { S:Ag Aux SV:APV O:Ptn Cpl }
$$

The verb "send" would have two valences which function as the subject by having the semantic role as an agent and as an object by having the semantic role as a patient. Those valences would be followed by additional information called as a complement. 
d) SEND in Transitive that is Manipulated with Ellipsis and Expansion Technique

In this part, the data of the English verb "send" will be analyzed by using ellipsis and expansion technique. The aim is to find how the verb "Send" can or cannot turn into another construction, and whether the sentence can be intransitive, transitive or ditransitive.

(13) When did you send it? BNC

(13a) When did you send it to me? (expansion)

(13b) When did you send? (ellipsis)

(14) It could send me to jail. BNC

(14a) It could send me a ticket to jail. (expansion)

(14b) It could send to jail. (ellipsis)

(15) All the publicity nearly sent him crazy. Ox

(15a) All the publicity nearly sent him. (ellipsis)

(15b) All the publicity nearly sent crazy. (ellipsis)

(16) Her musics always sends me to sleep. Ox

(16a) Her musics always sends me. (ellipsis)

(16b) Her musics always sends to sleep. (ellipsis)

(17) I will send them to the plane. BNC

(17a) I will send them (ellipsis)

(17b) I will send. (ellipsis)

(18) She sent the letter by airmail. Ox

(18a) She sent the letter. (ellipsis)

(18b) She sent. (ellipsis)

All the data above are some sentences in transitive construction that have been manipulated by ellipsis and expansion technique. Hence, there could be four conclusions coming from the explanation above. First, there is verb "send" in transitive construction that can turn into ditransitive construction as seen in data (13), and there is verb "send" in transitive construction that cannot turn into ditransitive construction as seen in data (14). Second, there is a verb "send" in transitive construction that must be followed by complement as seen in data (15), and there is verb "send" in intransitive construction with the addition of complement that can occur without the complement as the complement is not mandatory in the sentence as in data (18). Third, there is a verb "send" in transitive construction that must be followed by goal as seen in data (16), and there is a verb "send" in transitive construction with the addition of goal that can occur without the goal as the goal is not a mandatory in the sentence as seen in data (17). Last, there are no any of the transitive sentences shown in the example above could turn into intransitive sentences or construction as seen in data (28b), (29b), (30b), (31b), (32b), and (33b). The finding helps to prove the research statement above that the verb "send " as a single verb could not occur in intransitive construction.

\section{3) SEND in Ditransitive Construction}

Verb "send" in ditransitive construction will be analyzed in five different categorizations. First, verb "send' in ditransitive construction with pattern (Agent+Send+Patient+Recipient) Second, verb "send" in ditransitive construction with pattern (Agent+Send+Recipient+Patient). Third, Verb "send" in ditransitive construction with pattern (Agent+Send+Recipient+Patient+Goal). Fourth, verb send in ditransitive construction with pattern (Agent+Send+Recipient+Patient+Complement). Last, Verb "send" ditransitive construction with the manipulation of ellipsis and expansion technique.

\section{a) Agent+Send+Patient+Recipient}

The first construction in ditransitive construction has a pattern consist of (Agent+Send+Patient+Recipient).

(19) Have you sent a postcard to your mother yet? Ox

$$
\text { Aux S:Ag SV: APV DO: Ptn Prep IO: Rcp Prep }
$$

(20) I can send it to everyone. BNC

S: Ag Aux SV: APV DO: Ptn Prep IO:Rcp

(21) I will send them to Paul Rowlands. BNC

S: Ag Aux SV: APV DO: Ptn Prep IO:Rcp

The verb "send" would have three arguments. First, the argument will function as a subject with the semantic role as agent. Second, the argument will function as the direct object with the semantic 
role as a patient. Last, the argument will function as an indirect object with the semantic role as a recipient.

b) Agent+Send+Recipient+Patient

The grammatical construction in this ditransitive construction is (Agent+Send+Recipient+Patient).

(22) They will send you a certificate. BNC

S: Ag Aux SV: APV IO:Rcp DO:Ptn

(23) I will send you a text message. Ox

S: Ag Aux SV: APV IO:Rcp DO:Ptn

(24) We will send you $£$. BNC

S: Ag Aux SV: APV IO:Rep DO:Ptn

The verb "send" would have three arguments. First, the argument will function as a subject with the semantic role as agent. Second, the argument will function as the indirect object with the semantic role as a recipient. Last, the argument will function as a direct object with the semantic role as a patient.

c) Agent+Send+Recipient+Patient+Goal

The grammatical construction in this ditransitive construction is (Agent+Send+Recipient+Patient+Goal).

(25) You had to send them a planning application for it. BNC

S: Ag Aux SV: APV IO: Rcp DO: Ptn Goal

(26) I will send you one of the copies of the Agreement for your records. BNC

S: Ag Aux SV: PV IO: Rcp DO: Ptn Goal

(27) Those people send us a copy of their local Thompson Directory

S: Ag SV:APV IO: Rcp DO: Ptn

for getting in touch with local business. BNC

Goal

The verb "send" would have three arguments. First, the argument will function as a subject with the semantic role as agent. Second, the argument will function as the indirect object with the semantic role as a recipient. Last, the argument will function as a direct object with the semantic role as a patient. The sentence would also have an additional information that comes after both the objects called a goal.

\section{d) Agent+Send + Recipient + Patient + Complement}

The grammatical construction in this ditransitive construction is (Agent+Send+Recipient+Patient+Complement).

(28) We will send you a statement every April. BNC

$$
\text { S: Ag Aux SV: APV IO: Rcp DO: Ptn Cpl }
$$

(29) $\underline{\mathrm{We}}$ will send you an update itinerary. BNC

S: Ag Aux SV: APV IO: Rep DO: Ptn Cpl

(30) We will send you a new book automatically. BNC S: Ag Aux SV: APV IO: Rcp DO: Ptn Cpl

The verb "send" would have three arguments. First, the argument will function as a subject with the semantic role as agent. Second, the argument will function as the indirect object with the semantic role as a recipient. Last, the argument will function as a direct object with the semantic role as a patient. The sentence would also have an additional information that comes after both the objects called as a complement.

e) SEND in Ditransitive that is Manipulated with ellipsis and expansion technique

In this part, the data of the English verb "send" will be analyzed by using ellipsis and expansion technique. The aim is to find how the verb "Send" can or cannot turn into another construction, and whether the sentence can be intransitive, transitive or ditransitive.

(31) They will send you a certificate. BNC

(31a) They will send you. (ellipsis)

(31b) They will send. (ellipsis)

(32) I will send them to Paul Rowlands. BNC

(32a) I will send them. (ellipsis) 
(32b) I will send. (ellipsis)

(33) I can send it to everyone. BNC

(33a) I can send everyone it. (ellipsis)

(34) Have you sent a postcard to your mother yet? Ox

(34a) Have you sent your mother a postcard yet? (ellipsis)

(35) We will send you £5. BNC

(35a) We will send $£ 5$ for you. (expansion)

From the example of the ditransitive verb that has been manipulated by using ellipsis and expansion technique above, there can be found four conclusions. First, the English verb "send" in ditransitive construction could turn into transitive construction. The examples could be seen in data (31a) and (32a). Second, the English verb "send" in ditransitive construction could never be turned into intransitive construction as seen in data (31b) and (32b). Data (31b) and (32b) also help to prove that the English verb "send" would never be formed in intransitive construction. Third, some constructions of English verb "send" in ditransitive construction can turn into another construction as seen in data (34a) and (35a), and some constructions could not be seen in data (33a). The last, the prepositional phrase used as the indirect object or recipient in ditransitive construction could use a preposition "for" or "to" depending on the context. The example could be seen in data (50a).

\subsection{English Verb 'SEND' as a Phrasal Verb}

As a phrasal verb, the verb "send" will also be classified into three classifications. Verb "send" in intransitive construction, verb "send" in transitive construction, and verb "send" in ditransitive construction.

\section{1) SEND in Intransitive Construction}

The verb "send," as a phrasal verb would never occur in intransitive construction since English verb "send" as a phrasal verb would also need an object in its construction. Therefore, the verb "send" as a phrasal verb would always occur in transitive or ditransitive construction. Even if verb "send" as a phrasal verb could not occur in intransitive construction, there will be some cases where the verb " send" could seem to be in intransitive construction while they are not in intransitive construction. This phenomenon is not quite different from the data shown in data (1) and (2) above. The examples could be seen as follows:

(36) Beckham was sent off for a foul in the second half. Ox

$\begin{array}{lll}\text { S: Ptn } & \text { V: APV } & \text { Causer } \\ \text { (37) } & \text { make } & \text { it }\end{array}$

As seen in the data above, we can see the example of some sentences which appear in transitive construction but seems to be in intransitive construction which quite similar to data (1) and (2). What makes it different is just data (36) and (37) used verb "send" as a phrasal verb while data (1) and (12) used the verb "send" as a single verb.

\section{2) SEND in Transitive Construction}

Verb "send" as a phrasal verb in transitive construction, will be divided into four categories. First, the verb "send" as a phrasal verb in transitive construction with the pattern (Agent+Send+Patient). Second, the verb "send" as a phrasal verb in transitive construction with the pattern (Agent+Send+Patient+Goal). Third, verb "send" in transitive construction with the pattern (Agent+Send + Patient + Complement). Last, verb "send" as a phrasal verb in transitive construction that is manipulated with ellipsis and expansion technique.

a) Agent+Send+Patient

The first grammatical construction of the English verb "send" in the form of a phrasal verb has the pattern of (Agent+Send+Patient).

(38) She sent for the latest sales figures. Ox

$$
\text { S: Ag PV: APV O: Ptn }
$$

(39) $\underline{\text { She }}$ will send her best men down. BNC

S: Ag Aux PV: APV O: Ptn PV: APV

(40) $\underline{\mathrm{We}}$ will send in a Trojan horse. BNC

$$
\text { S: Ag Aux PV: APV O: Ptn }
$$

The phrasal verb would need two arguments in the construction. First, the argument will function as a subject with the semantic role as agent. Second, the argument will function as an object with the semantic role of the patient. 
b) Agent+Send+Patient+Goal

The next construction of the English verb "send" in the form of a phrasal verb has the pattern of (Agent+Send+Patient+Goal).

(41)I have sent off for some books for my course. Ox S: Ag Aux PV: APV O: Ptn Goal

(42) We send them away to school. BNC

S: Ag PV: APV O: Ptn PV: APV Goal

(43)I will send for Jim Kielthy to clear up this mess. BNC

S: Ag Aux PV: APV O: Ptn Goal

The phrasal verb would need two arguments in the construction. First, the argument will function as a subject with the semantic role as agent. Second, the argument will function as an object with the semantic role of the patient. Then, at the end of the sentence, there would be additional information which would have a semantic role as a goal.

c) Agent+Send+Patient+Complement

The other construction of the English verb "send" in the form of a phrasal verb has the pattern of (Agent+Send+Patient+Complement).

(44) We sent our furniture on by ship. Ox

S: Ag PV: APV O:Ptn PV:APV Cpl

(45) $\underline{\text { Mel }}$ will send up the ex-Mirror publisher in his TV show. BNC

S: Ag Aux PV: APV O:Ptn Cpl

(46) They will send out statements in their own name. BNC

S: Ag Aux PV: APV O:Ptn Cpl

The phrasal verb would need two arguments in the construction. First, the argument will function as a subject with the semantic role as agent. Second, the argument will function as an object with the semantic role of a patient. Those arguments would be followed by additional information called a complement.

d) SEND as Phrasal Verb in Transitive Construction that is Manipulated with Ellipsis and Expansion Technique

In this part, we will analyze the English verb "send" in the form of a phrasal verb by using ellipsis and expansion technique. It aims to find out how English verb "send" as a phrasal verb can or cannot turn into another construction, and whether the construction can be in the form of intransitive, transitive or ditransitive.

(47) Send the nurse in. BNC

(47a) Send the nurse in to the patient. (expansion)

(47b) Send in. (ellipsis)

(48) I must send out the invitation. BNC

(48a) I must send out the invitation to the guest. (expansion)

(48b) I must send out. (ellipsis)

From the data above, there could be found two conclusions from the example of phrasal verb "send" that is manipulated with ellipsis and expansion technique. First, it could be seen that there is phrasal verb "send" in transitive construction that could turn into ditransitive construction as seen in data (47a), and there is a phrasal verb "send" in transitive construction that could not turn into ditransitive construction as seen in data (48a). Second, phrasal verb "send" in transitive construction cannot turn into intransitive construction as seen in data (47b) and (48b). The data help to prove the statement that the English verb "send" both as a phrasal verb or single verb could not turn into intransitive construction.

\section{3) SEND in Ditransitive Construction}

Verb "send" as a phrasal verb in ditransitive construction in this research will only be divided into two categories. First, the verb "send" as a phrasal verb in ditransitive construction with the pattern (Agent+Send+Patient+Recipient). Second, the verb "send" as a phrasal verb in ditransitive construction that is manipulated with ellipsis and expansion technique.

a) Agent+Send+Patient+Recipient

The first construction of the verb "send" as a phrasal verb in ditransitive construction has a pattern consist of (Agent+Send+Patient+Recipient). 
(49) Its use would send out the wrong signal to consumers. BNC

S: Ag Aux PV: APV DO: Ptn Prep IO: Rcp

(50) The Queen send out a search party for her husband. BNC S: Ag PV: APV DO: Ptn Prep IO: Rep

(51) I am sending the files off to my boss. Ox
S: Ag PV: APV DO: Ptn PV: APV Prep IO: Rcp

The verb "send" would have three arguments. First, the argument will function as a subject with the semantic role as agent. Second, the argument will function as the direct object with the semantic role as a patient. Last, the argument will function as an indirect object with the semantic role as a recipient.

\section{b) SEND as Phrasal Verb in Ditransitive Construction That is Manipulated with Ellipsis and Expansion Technique}

In this part, we will analyze the of the English verb "send" as a phrasal verb in ditransitive construction by using ellipsis and expansion technique. It aims to find out how English verb "send" as a phrasal verb can or cannot turn into another construction, and whether the construction can be in the form of intransitive, transitive or ditransitive.

(52) Its use would send out the wrong signal to consumers. BNC

(52a) Its use would send out the wrong signal. (ellipsis)

(52b) Its use would send out. (ellipsis)

(53) I am sending the files off to my boss. Ox

(53a) I am sending my boss the files off. (ellipsis)

(53b) I am sending off my boss the files. (ellipsis)

(53c) I am sending the files off to my boss for the meeting. (expansion)

(53d) I am sending the files off to my boss tomorrow. (expansion)

From the data above, there can be found five conclusions. First, phrasal verb "send" in ditransitive construction can turn into transitive construction as seen in data (52a) Second, phrasal verb "send" in ditransitive construction could not turn into intransitive construction. Data (52b) is another prove that support the statement that verb "send" as phrasal or single would never turn into intransitive construction. Third, phrasal verb "send" in ditransitive construction could not appear with pattern (Agent+Send+Recipient+Patient), but rather appear with the pattern (Agent+Send+Patient+Recipient) even if with the addition of complement or goal. The example can be seen in data (53a) and (53b). Fourth, phrasal verb "send" in ditransitive construction could have an additional information which is a goal as in data (53c). So, it could have the construction with pattern (Agent+Send+Patient+Recipient+Goal). Fifth, phrasal verb "send" in ditransitive construction could also have an additional information called as a complement as in data (53d). So, it could also have the construction with pattern (Agent+Send+Patient+Recipient+Complement).

\section{Conclusion}

English verb "send" could have 2 to 3 arguments with the semantic role as Agent, Patient, and Recipient. English verb "send" could appear in transitive construction and ditransitive construction, but would never appear in intransitive construction. There might be some sentences of the English verb "send" which seems to be in intransitive construction, but they are actually not. Those phenomena could be found if the English verb "send" both as single or phrasal appear as passive voice and to-infinitive.

In the form of a single verb, from the data found in Oxford Advanced Learner's Dictionary and British National Corpus, the English verb "send' in transitive construction could be found in three different constructions. (i) [Agent + Send + Patient]; (ii) [Agent + Send + Patient + Goal]; (iii) [Agent + Send + Patient + Complement]. Then, the English verb "send' in ditransitive construction could be found in four different construction. (i) [Agent + Send + Patient + Recipient]; (ii) [Agent+Send+Recipient+Patient]; (iii) [Agent + Send + Recipient + Patient + Goal]; (iv) (Agent + Send + Recipient + Patient + Complement). There could be found some additional findings after some data of the English verb "send" as a single verb are manipulated with ellipsis and expansion technique, (i) there is verb "send" in transitive construction that can turn into ditransitive and there is verb "send" in transitive construction that cannot turn into ditransitive construction; (ii) there is verb 
"send" in transitive construction that must be followed by complement and there is verb "send" in intransitive construction with the addition of complement that can occur without the complement as the complement is not a mandatory; (iii) there is verb "send" in transitive construction that must be followed by goal, and there is verb "send" in transitive construction with the addition of goal that can occur without the goal as the goal is not a mandatory; (iv) English verb "send" in ditransitive construction could turn into transitive construction; (v) some constructions of English verb "send" in ditransitive construction can turn into another construction, and some constructions could not; (vi) the prepositional phrase used as the indirect object or recipient in ditransitive construction could use a preposition "for" or "to" depending on the context.

In the form of phrasal verb, from the data found in Oxford Advanced Learner's Dictionary and British National Corpus, English verb "send" in transitive construction could be found in three different constructions. (i) [Agent + Send + Patient]; (ii) $[$ Agent + Send + Patient + Goal]; (iii) [Agent + Send + Patient + Complement]. Then, English verb "send" in ditransitive construction could only be found in one construction which [Agent + Send + Patient + Recipient]. There could also be found some additional findings after some data of English verb "send" as phrasal verb are manipulated with ellipsis and expansion technique; (i) there is phrasal verb "send" in transitive construction that could turn into ditransitive construction, and there is phrasal verb "send" in transitive construction that could not turn into ditransitive construction; (ii) phrasal verb "send" in ditransitive construction can turn into transitive construction; (iii) phrasal verb "send" in ditransitive construction could not appear with pattern [Agent + Send + Recipient + Patient]; (iv) phrasal verb "send" in ditransitive construction could have an additional information which is goal with pattern [Agent + Send + Patient + Recipient + Goal]; (v) phrasal verb "send" in ditransitive construction could also have an additional information called as complement with pattern [Agent + Send + Patient + Recipient + Complement].

\section{Acknowledgment}

Our sincere gratitude is hereby extended to the head of English Education Department of Universitas Ahmad Dahlan and dearest participants who never cease in helping until this research is accomplished.

\section{References}

Chafe, W.L. (1970). Meaning and the structure of language. Chicago: University of Chicago Press.

Evans, V., \& Green, M. (2006). Cognitive linguistics: An introduction. Edinburgh: Edinburgh University Press.

Gisborne, N. (2010). The event structure of perception verbs. New York: Oxford University Press

Goldberg, A.E. (1995). A construction grammar approach to argument structure. Chicago: The University of Chicago Press.

Jackendoff, R. (2003). Foundations of language: Brain, meaning, grammar, evolution. New York: Oxford University Press Inc.

Lakoff, G. (2007). Cognitive models and prototype theory. In: The cognitive linguistics reader. Ed. Evans, Vyvyan., Bergen, K. Benjamin, Zinken, Jörg. London: Equinox Publishing, Ltd.

Moyer, M.G. \& Wei, L. (2008). The blackwell guide to research methods in bilingualism and multilingualism. Oxford: Blackwell.

Prayudha. (2014). Polisemi pada verba LOOK: Sebuah kajian linguistik kognitif. (S2 Thesis). Yogyakarta: Universitas Gadjah Mada

Sudaryanto. (2005). Metode dan aneka teknik analisis bahasa. Yogyakarta: Sanata Dharma University Press.

Tavakoli, H. (2002). A dictionary of research methodology and statistics in applied linguistics. Rahnama Press.

Van Valin, Robert D. jr. (2005). Exploring the syntax-semantics interface. Cambridge: MIT Press.

Verhaar, J.W.M. (2010). Asas-asas linguistic umum. Yogyakarta: Gadjah Mada University Press 